\title{
Correction to: An analysis of managerial delegation in a market with vertically-integrated producer owning an essential input monopolistically
}

\section{Chung-Hui Chou ${ }^{1}$ (D}

Published online: 20 January 2022

(c) Springer-Verlag GmbH Germany, part of Springer Nature 2022

\section{Correction to: Review of Economic Design}

$$
\text { https://doi.org/10.1007/s10058-021-00274-3 }
$$

In Appendix 2, the last mathematical expression

$$
\begin{aligned}
& s w^{*}\left(\lambda_{1}^{*}, \lambda_{2}^{*}\right)-s w^{*}(1,1) \\
& =\frac{\left(c_{2}-c_{1}\right)\left(880 a-1591 c_{1}+711 c_{2}\right)}{12800}\left\{\begin{array}{r}
>0 \text { if } a<\frac{1591 c_{1}-711 c_{2}}{880} \\
\langle 0 \text { if } a\rangle \frac{1591 c_{1}-711 c_{2}}{880}
\end{array}\right.
\end{aligned}
$$

should be

$$
\begin{aligned}
& s w^{*}\left(\lambda_{1}^{*}, \lambda_{2}^{*}\right)-s w^{*}(1,1) \\
& =\frac{\left(c_{2}-c_{1}\right)\left(880 a-1591 c_{1}+711 c_{2}\right)}{12800}\left\{\begin{array}{l}
>0 \text { if } a<\frac{1591 c_{1}-711 c_{2}}{880} \\
<0 \text { if } a>\frac{1591 c_{1}-711 c_{2}}{880}
\end{array}\right.
\end{aligned}
$$

In Appendix 5, the last mathematical expression

$$
\left|\frac{d \pi_{1}^{*}\left(\lambda_{1}^{*}, \lambda_{2}^{*}\right)}{d c_{1}}\right|-\left|\frac{d \pi_{2}^{*}\left(\lambda_{1}^{*}, \lambda_{2}^{*}\right)}{d c_{2}}\right|=\frac{64 a-175 c_{1}+111 c_{2}}{128}\left\{\begin{array}{l}
<0 \text { if } a<\frac{175 c_{1}-111 c_{2}}{64} \\
>0 \text { if } a>\frac{175 c_{1}-111 c_{2}}{64}
\end{array} .\right.
$$

The original article can be found online at https://doi.org/10.1007/s10058-021-00274-3.

$凶$ Chung-Hui Chou

chchou@isu.edu.tw

1 Department of Finance, I-Shou University, Kaohsiung City 84001, Taiwan 


\section{should be}

$$
\left|\frac{d \pi_{1}^{*}\left(\lambda_{1}^{*}, \lambda_{2}^{*}\right)}{d c_{1}}\right|-\left|\frac{d \pi_{2}^{*}\left(\lambda_{1}^{*}, \lambda_{2}^{*}\right)}{d c_{2}}\right|=\frac{64 a-175 c_{1}+111 c_{2}}{128}\left\{\begin{array}{l}
<0 \text { if } a<\frac{175 c_{1}-111 c_{2}}{64} \\
>0 \text { if } a>\frac{175 c_{1}-111 c_{2}}{64}
\end{array}\right.
$$

Publisher's Note Springer Nature remains neutral with regard to jurisdictional claims in published maps and institutional affiliations. 